\title{
Vacinação contra a influenza autorreferida por idosos de áreas rurais ribeirinhas: implicação potencial dos achados frente à pandemia de covid-19 no Amazonas
}

Influenza vaccination in older adults living in rural riverside areas: potential implication of the findings regarding the covid-19 pandemic in Amazonas

\author{
Anny Beatriz Costa Antony de Andradel (ID) \\ Bernardino Cláudio de Albuquerque $\mathbb{1}$ \\ Luiza Garnelo' $\mathbb{I D}^{2}$ \\ Fernando José Herkrath',2 $\mathbb{I D}$
}

\section{Resumo}

Objetivo: Avaliar a proporção de pessoas idosas não vacinadas e os motivos que interferem na imunização contra influenza em localidades rurais ribeirinhas, discutindo as potenciais implicações na vacinação contra a infecção por SARS-CoV-2. Método: Inquérito epidemiológico de base domiciliar realizado em 38 localidades rurais ribeirinhas de Manaus (AM), Brasil, assistidas por uma Unidade Básica de Saúde Fluvial. Os participantes responderam a um questionário que investigou condições de vida, saúde e acesso aos serviços de saúde. Para este estudo foram avaliados os desfechos relacionados à imunização contra a Influenza nos últimos 12 meses e o principal motivo alegado para a não vacinação. Foi realizada análise descritiva dos dados, seguida por análise de regressão logística para identificar fatores associados à não vacinação. Resultados: Das 102 pessoas idosas incluídas no estudo, 28 (27,5\%) referiram não vacinação contra a Influenza no ano anterior. Os principais motivos foram a falta de informação sobre a vacinação $(60,7 \%)$ e barreiras de acesso aos serviços de saúde (28,6\%). Foi identificada maior chance de não vacinação entre aqueles que não consultaram o médico no último ano $(\mathrm{RC}=4,18$; $\mathrm{IC} 95 \%=1,57$ 11,11) e com maior renda domiciliar $(\mathrm{RC}=1,08$; IC95\%=1,02-1,14). Conclusão: Verificou-se elevada proporção de pessoas idosas autorreferindo não imunização contra Influenza. Os motivos identificados para a não vacinação podem também representar barreiras à vacinação desse grupo populacional contra a covid-19. Dessa forma, faz-se necessário adequar o planejamento da vacinação em contextos rurais ribeirinhos, desenvolvendo estratégias mais contextualizadas para garantia de cobertura a essa população com maior vulnerabilidade aos efeitos de doenças respiratórias.

\footnotetext{
Fundação Oswaldo Cruz, Instituto Leônidas e Maria Deane, Laboratório de Situação de Saúde e Gestão do Cuidado de Populações Indígenas e Outros Grupos Vulneráveis. Manaus, AM, Brasil.

2 Universidade do Estado do Amazonas, Escola Superior de Ciências da Saúde. Manaus, AM, Brasil.
}

Financiamento da pesquisa: Programa de Pesquisa para o SUS (PPSUS)/ Fundação de Amparo à Pesquisa no Amazonas (FAPEAM) chamada n001/2017; Chamada Nº01/2020 - PROEP-LABS/ILMD Fiocruz Amazônia.

Os autores declaram não haver conflito na concepção deste trabalho.

Palavras-chave: População rural. Cobertura Vacinal. Saúde do Idoso. Vacinas contra Influenza. 


\section{Abstract}

Objective: To evaluate the proportion of unvaccinated older adults and the reasons that interfere with immunization against Influenza in rural riverside locations, discussing the potential implications on vaccination against SARS-CoV-2 infection. Method: Householdbased survey conducted in 38 rural riverside locations in Manaus, Amazonas, Brazil, covered by a primary care Fluvial Health Unit. Participants answered a questionnaire that investigated living conditions, health status and access to health services. In this study, the outcomes related to immunization against Influenza in the last 12 months and the main reported reason for non-vaccination were evaluated. Descriptive data analysis was performed, followed by logistic regression to identify factors associated with non-vaccination. Results: Of the 102 older adults included in the study, 28 (27.5\%) reported not vaccinating against Influenza in the previous year. The main reasons were lack of information about vaccination $(60.7 \%)$ and barriers to accessing health services (28.6\%). An increased chance of non-vaccination was identified among those who did not see a doctor in the last year $(\mathrm{OR}=4.18 ; 95 \% \mathrm{CI}=1.57-11.11)$ and those with higher household income $(\mathrm{OR}=1.08 ; 95 \% \mathrm{CI}=1.02-1.14)$. Conclusion: A high proportion of older adults reporting no immunization against Influenza was identified. The reasons for non-vaccination may also represent barriers to the vaccination of this population group against COVID-19. Thus, it is necessary to improve the vaccination planning in rural riverside contexts, developing more contextualized strategies to assure coverage for this population, more vulnerable to the effects of respiratory diseases.
Keywords: Rural Population. Vaccination Coverage. Health of the Elderly. Influenza Vaccines.

\section{INTRODUÇão}

Desigualdades sociais e em saúde são marcantes no território brasileiro ${ }^{1}$, em especial entre as populações rurais, caracterizadas pelas piores condições de vida e de saúde, por barreiras geográficas e financeiras no acesso aos serviços, insuficiência de profissionais de saúde e frágil estrutura assistencial ${ }^{2}$.

Além de mais vulneráveis a tais iniquidades, as pessoas idosas compõem o grupo populacional sob maior risco de doenças respiratórias e sistêmicas ${ }^{3}$. A pandemia da covid-19 acentuou seus riscos à saúde, inserindo-os como grupo prioritário para a vacinação, enquanto estratégia para o controle dos impactos da epidemia ${ }^{4}$. Neste contexto, refletir sobre os principais motivos que dificultam a vacinação desse grupo contra a Influenza se mostra relevante, uma vez que tais motivações poderiam também representar entraves à vacinação contra a covid-19.

Assim, o presente estudo teve como objetivo avaliar a proporção de pessoas idosas não vacinadas e os motivos que interferem na imunização contra a Influenza em localidades rurais ribeirinhas, discutindo as potenciais implicações na vacinação contra a infecção por SARS-CoV-2.

\section{MÉTODO}

Inquérito epidemiológico de base domiciliar, realizado no ano de 2019 em 38 localidades rurais ribeirinhas da margem esquerda do rio Negro, Manaus (AM), Brasil, situadas na área de abrangência de uma unidade básica de saúde fluvial (UBSF) do município. Para o estudo foi realizada amostragem aleatória estratificada, calculada com base no número de indivíduos e domicílios informados pelos agentes comunitários de saúde que atuavam no território. A seleção dos domicílios em cada localidade foi aleatória sistemática. O cálculo do tamanho da amostra considerou a probabilidade de encontrar os grupos de interesse do inquérito nos domicílios, entre eles pessoas idosas, com idade de 60 anos ou mais ( $p=0,118$ ), uma prevalência de $50 \%$ dos desfechos de interesse e precisão de $95 \%$, sendo acrescentado $10 \%$ para possíveis perdas ou recusas, com ajuste para a população finita ( $\mathrm{N}=277$ ), tendo sido visitados 287 de um universo de 765 domicílios dispersos ao longo do território. As localidades ribeirinhas estudadas são acessíveis apenas por via fluvial, levando cerca de 12 horas o deslocamento regular entre a área urbana do município e a localidade mais distante da região pesquisada. 
As pessoas idosas selecionadas responderam a um questionário desenvolvido no aplicativo Research Electronic Data Capture (REDCap) referentes a questões socioeconômicas, relacionadas à saúde e acesso aos serviços de saúde. O desfecho principal do estudo foi avaliado através da questão "Nos últimos 12 meses, tomou vacina contra gripe?" Para aqueles que não tinham sido imunizados, questionou-se através de pergunta aberta o principal motivo para a não vacinação. As respostas dos idosos referentes aos motivos para não vacinação foram analisadas e classificadas em três categorias, definidas após análise: 1-Fragilidades das informações a respeito da vacinação, 2-Barreiras de acesso à vacinação e 3-Outros motivos especificados.

Após verificação das inconsistências, os dados foram exportados para a análise descritiva das variáveis. Em seguida, realizou-se análise de regressão logística entre as variáveis independentes e o desfecho de não vacinação, estimando-se as razões de chance ajustadas (RC) e respectivos intervalos de confiança a $95 \%$. As variáveis incluídas na análise múltipla foram sexo, idade, logaritmo da renda domiciliar (em R $\$$ ), já ter frequentado escola, autopercepção da saúde e referir consulta médica nos últimos 12 meses.

O presente estudo é vinculado ao projeto Estudo exploratório das condições de vida, saúde e acesso aos serviços de saúde depopulações rurais ribeirinhas de Manaus e Novo Airão, Amazonas, aprovado pelo Comitê de Ética em Pesquisa envolvendo Seres Humanos, sob o parecer no 1.742.086.

\section{RESULTADOS}

Nos 287 domicílios visitados foram avaliadas 102 pessoas idosas, 56 do sexo masculino $(54,9 \%)$ e 46 do sexo feminino (45,1\%). A média de idade foi 67,8 $( \pm \mathrm{dp}=8,1)$, variando de 60 a 90 anos. Nos domicílios com pessoas idosas, o número médio de moradores foi 3,2 (mínimo=1, máximo=9). A renda domiciliar mensal média foi $\mathrm{R} \$ 1.600,17( \pm \mathrm{dp}=836,19)$ e 44 $(43,1 \%)$ entrevistados nunca tinham frequentado escola. Dentre os idosos, $28(27,5 \%)$ não foram vacinados contra a Influenza no ano anterior (IC95\%: 19,6-37,1). A Tabela 1 apresenta os principais motivos referidos pelos idosos para a não vacinação.

Tabela 1. Motivos relacionados à não vacinação contra a Influenza em pessoas idosas residentes em localidades rurais ribeirinhas, Manaus, Amazonas, 2019.

\begin{tabular}{ll}
\hline Principal motivo para a não vacinação contra a Influenza & $\mathrm{n}=28(\%)$ \\
\hline Fragilidade das informações a respeito da vacinação & $2(7,14)$ \\
Raramente é acometido por gripe & $3(10,72)$ \\
Não sabia que era necessário vacinar-se & $1(3,57)$ \\
Não sabia onde vacinar-se & $4(14,29)$ \\
Medo de reações adversas da vacina & $1(3,57)$ \\
Tem medo da injeção & $3(10,72)$ \\
Não acredita que a vacina protege & $1(3,57)$ \\
Estava gripado durante a campanha de vacinação, porém não buscou vacinar-se quando sadio & $1(3,57)$ \\
Não possuir cartão de vacina & $1(3,57)$ \\
Não soube informar & $1(3,57)$ \\
Barreiras de acesso à vacinação & $2(7,14)$ \\
Curto espaço de tempo para a oferta da vacina & $1(3,57)$ \\
Ausência de acompanhante para deslocamento ao serviço & $3(10,72)$ \\
Não foi oferecida no serviço & $1(3,57)$ \\
Não estar presente na comunidade no momento da vacinação & $1(3,57)$ \\
Ausência de recurso para combustível & $1(3,57)$ \\
\hline Outros motivos especificados & $1(3,57)$ \\
Contraindicação médica & \\
Não consentiu na vacinação & \\
Referiu alergia à vacina &
\end{tabular}

Fonte: dados da pesquisa, 2021. 
A maioria dos motivos especificados pelos idosos para a não vacinação foram as fragilidades das informações sobre a vacinação $(60,71 \%)$, seguido por motivos relacionados às barreiras de acesso à vacinação (28,58\%). A análise de regressão mostrou maior chance de não vacinação entre os idosos que não consultaram o médico no último ano $(\mathrm{RC}=3,75$; IC95\%=1,41-9,96) e entre aqueles com maior renda domiciliar $(\mathrm{RC}=3,03$; IC $95 \%=1,18$ $7,79)$. Não foi encontrada associação com sexo $(\mathrm{RC}=1,01 ; \mathrm{IC} 95 \%=0,39-2,67)$, idade $(\mathrm{RC}=0,99$; $\mathrm{IC} 95 \%=0,94-1,06)$, ter frequentado escola $(\mathrm{RC}=0,63$; IC95\%=0,23-1,72) e autopercepção da saúde $(\mathrm{RC}=1,04 ; \mathrm{IC} 95 \%=0,74-1,46)$.

\section{DISCUSSÃO}

É preocupante o percentual de idosos não vacinados contra a Influenza que residem nas localidades rurais ribeirinhas estudadas. Os achados sugerem que a não vacinação dos idosos é multicausal, envolvendo tanto falhas no processo de informação a respeito da importância da vacina, quanto barreiras de acesso ao serviço de saúde. As motivações encontradas podem não se limitar à vacinação contra a Influenza, mas representarem impeditivos também à cobertura vacinal contra a covid-19 no território em questão.

A educação em saúde disponibilizada ao usuário é fundamental no esclarecimento sobre a importância da vacinação na prevenção de doenças, os potenciais efeitos adversos e para a desmistificar informações equivocadas a respeito do processo vacinal $l^{5}$. Nesse sentido, os achados sugerem que os indivíduos com maior renda domiciliar representam um público-alvo importante para as ações que visem ampliar a cobertura vacinal. A ocorrência de efeitos adversos e o descrédito à vacinação são reportadas como importantes causas de evasão de idosos nas campanhas de vacinação contra Influenza ${ }^{6}, \mathrm{com}$ potencialidade para também interferir negativamente na campanha de vacinação contra a covid-19.

É necessário que os planos de vacinação considerem as barreiras geográficas presentes no contexto rural ribeirinho e promovam estratégias para ampliação do acesso, não se limitando a reproduzir o planejamento adotado nos centros urbanos ${ }^{7}$. Tais medidas podem garantir uma adequada cobertura vacinal, principalmente entre os idosos, que representam uma parcela significativa da população rural, dado o êxodo seletivo de jovens ${ }^{8}$, além de constituírem o grupo considerado de maior risco para mortalidade por doenças respiratórias como a covid- $19^{4}$.

Por residir no território e atuar como elo de ligação com o serviço, o agente comunitário de saúde é um ator fundamental nas ações de educação em saúde centradas na comunidade. Também tem papel relevante na busca ativa de idosos não imunizados e no reconhecimento do território, no repasse de informações e discussão de estratégias de vacinação com os demais componentes da equipe de saúde". O acesso regular ao serviço de atenção primária à saúde é também uma estratégia importante para a vacinação, sendo que os idosos que relataram não terem consulta médica nos últimos 12 meses tiveram quase quatro vezes mais chance de também não terem se vacinado contra a Influenza. A organização operacional da UBSF pode ser maximizada de modo a ampliar as oportunidades de vacina, bem como promover pactuações prévias da equipe com a comunidade, visando contribuir para o aumento da cobertura vacinal. Além da adequada organização dos serviços, a provisão de informação adequada aos usuários em relação à necessidade de retorno para as doses anuais, ou segunda dose vacinal como no caso da covid-19, é primordial para que a cobertura seja atingida adequadamente.

Reitera-se que a baixa cobertura vacinal pode contribuir para a mutação viral e consequente resistência das variantes às vacinas ${ }^{10}$, aumento no número de casos e de óbitos na população, principalmente nos grupos mais vulneráveis, como os idosos. O estudo apresenta como limitação o uso de informações referidas, sujeitas a vieses de informação. Considerando o desenho transversal do estudo, as inferências causais baseadas nas medidas de associação também devem ser interpretadas com cautela. Embora a intenção do estudo seja provocar uma reflexão crítica a partir dos achados referentes à vacinação contra a Influenza, de forma a contribuir 
com a organização dos serviços diante da realidade imposta pela pandemia da covid-19, o comportamento relacionado aos dois agravos pode não ser similar.

\section{CONCLUSÃO}

O presente estudo analisou os motivos relacionados à não vacinação contra a Influenza em idosos residentes em localidades rurais ribeirinhas, em um contexto de premência na vacinação contra a covid-19. Além das dificuldades enfrentadas pela população relacionadas às barreiras de acesso aos serviços, foram observadas fragilidades na disponibilidade eficaz de informações a respeito da vacinação entre os idosos. A garantia e ampliação da cobertura vacinal de populações rurais ribeirinhas demanda um planejamento estratégico específico que contemple a competência cultural e os modos de vida e as situações de vulnerabilidade, permitindo maior equidade no acesso aos serviços e ações em saúde.

Editado por: Maria Helena Rodrigues Galvão

\section{REFERÊNCIAS}

1. Albuquerque MV, Viana ALD, Lima LD, Ferreira MP, Fusaro ER, Iozzi FL. Desigualdades regionais na saúde: mudanças observadas no Brasil de 2000 a 2016. Ciênc Saúde Colet. 2017;22(4):1055-64. Disponível em: https://doi.org/10.1590/141381232017224.26862016.

2. Garnelo L, Lima JG, Rocha ESC, Herkrath FJ. Acesso e cobertura da Atenção Primária à Saúde para populações rurais e urbanas na região norte do Brasil. Saúde Debate. 2018;42(spe1):81-99. Disponível em: https://doi.org/10.1590/0103-11042018S106.

3. Center for Disease Control and Prevention [Internet]. USA: CDC; 1946. COVID-19 - Older Adults: At greater risk of requiring hospitalization or dying if diagnosed with COVID-19; 2021 [acesso em 14 abr. 2021]; [1 tela]. Disponível em: https://www.cdc.gov/coronavirus/2019ncov/need-extra-precautions/older-adults.html.

4. Souto EP, Kabad J. Hesitação vacinal e os desafios para enfrentamento da pandemia de COVID-19 em idosos no Brasil. Rev Bras Geriatr Gerontol. 2020;23(5):e210032. Disponível em: https://doi. org/10.1590/1981-22562020023.210032.

5. Silva SPC, Menandro MCS. Representações de idosos sobre a vacina da gripe. Ciênc Saúde Colet. 2013;18(8):2179-88. Disponível em: https://doi. org/10.1590/S1413-81232013000800002.
6. Gonçalves AR, Nogueira PC. Vacinação contra influenza para idosos: motivos da não adesão. Geriatr Gerontol Aging. 2013;7:142-5. Disponível em: https:// cdn.publisher.gn1.link/ggaging.com/pdf/v7n2a10.

7. Garnelo L, Parente RCP, Pucciarelli MLR, Correia PC, Torres MV, Herkrath FJ. Barriers to access and organization of primary health care services for rural riverside populations in the Amazon. Int J Equity Health. 2020;19(1):1-10. Disponível em: https://doi. org/10.1186/s12939-020-01171-x.

8. Pessoa VM, Almeida MM, Carneiro FF. Como garantir o direito à saúde para as populações do campo, da floresta e das águas no Brasil? Saúde Debate. 2018;42(Esp. 1):302-14. Disponível em: https://doi.org/10.1590/0103-11042018S120.

9. dos Santos MDM, Cazola LHO. Adesão à vacina de influenza na área urbana de Aquidauana-MS coberta pelo Programa Saúde da Família. Epidemiol Serv Saúde. 2008;17(2):145-8. Disponível em: http://dx.doi. org/10.5123/S1679-49742008000200013.

10. Houser K, Subbarao K. Influenza vaccines: challenges and solutions. Cell Host Microbe. 2015;17(3):295-300. Disponível em: http://dx.doi. org/10.1016/j.chom.2015.02.012. 